\title{
An actuated larynx phantom for pre-clinical evaluation of droplet-based reflex- stimulating laryngoscopes
}

Jacob Friedemann Fast, Leibniz Universität Hannover, Institute of Mechatronic Systems, Hannover, Germany, jacob.fast@imes.uni-hannover.de

Jiazhen He, Leibniz Universität Hannover, Institute of Mechatronic Systems, Hannover, Germany

Michael Jungheim, Hannover Medical School, Department of Phoniatrics and Pediatric Audiology, Hannover, Germany Tobias Ortmaier, Leibniz Universität Hannover, Institute of Mechatronic Systems, Hannover, Germany

Martin Ptok, Hannover Medical School, Department of Phoniatrics and Pediatric Audiology, Hannover, Germany

Lüder Alexander Kahrs, Center for Image Guided Innovation and Therapeutic Intervention (CIGITI), The Hospital for Sick Children, Toronto, Canada \& Department of Mathematical and Computational Sciences, University of Toronto Mississauga, Mississauga, Canada

The laryngeal adductor reflex (LAR) is an important protective function of the larynx to prevent aspiration and subsequently, potentially fatal aspiration pneumonia by rapidly closing the glottis. Recently, a novel method for targeted stimulation and evaluation of the LAR has been proposed to enable non-invasive and reproducible LAR performance grading and to extend the understanding of this reflexive mechanism. The method relies on the laryngoscopically controlled application of accelerated water droplets in association with a high-speed camera system for LAR stimulation site and reflex onset latency identification. In recent works, this device has been enhanced by adding stereoscopic vision and a mechatronic system for droplet formation control. Prior to extensive clinical trials, an experimental testing of prototype devices in a lab setting is highly desired. Furthermore, a demonstration of the method using a realistic phantom could increase patient compliance in a future clinical setting. For these purposes, a model of the human larynx including vocal fold adduction capabilities for LAR simulation was developed in this work. The combination of near real-time image processing based on a custom algorithm and individual motorization of each vocal fold enables spatio-temporal droplet impact detection and controlled vocal fold adduction. To simulate different LAR pathologies, the current implementation allows to individually adjust the reflex onset latency of the ipsi- and contralateral vocal fold with respect to the automatically detected impact location of the droplet as well as the maximum adduction angle of each vocal fold. An experimental study of the temporal offset between desired and observed LAR onset latency due to image data processing was performed based on high-speed recordings of the model reaction to compensate this factor. In the future, alternative methods for droplet impact detection could be explored and droplet impact intensity measurement capabilities could be added to the assembly presented here. 


\section{Questionnaire to evaluate user acceptance before purchasing medical devices in health facilities}

Lorenz Müller, Fachhochschule Münster, Steinfurt, Germany, lorenz.mueller@fh-muenster.de Claus Backhaus, Fachhochschule Münster, Steinfurt, Germany, claus.backhaus@fh-muenster.de

Ergonomically poorly designed medical devices disturb clinical processes. This results in increased burdens on medical staff and reduced patient safety. Nevertheless, purchasing decisions are driven by comparable values like technical specifications and prices rather than usability. Even if medical devices are tested to gather user opinions, these trials are usually not evaluated in a standardized way. The aim of the study is to develop a standardized questionnaire, which evaluates the user acceptance of medical devices during in-house product trials in procurement processes in healthcare facilities. For this purpose, procurement processes were participative analysed with 69 employees from 63 health facilities. As part of the process analysis, a semi-structured interview was conducted to identify product requirements. The openly asked product requirements were clustered by using inductive category formation. The questionnaire items were derived from interview statements as well as usability questionnaires and sorted into the identified categories. The developed questionnaire was used in five simulated product tests and a usability study. The internal consistency was tested using Cronbach's Alpha. For validation the correlation between the questionnaire result and the perceived product satisfaction, which was additionally asked using a seven-point adjective-anchored Likert scale, was analysed. Out of 249 product requirements, eleven categories were identified like functionality, usability, workflow, compatibility, product quality or mobility. The questionnaire includes 25 closed items, which evaluate the identified categories using a five-point Likert scale. Of these, 13 items are fixed. To address specific requirements, the remaining 12 items can be added variably. The internal consistency of the questionnaire is excellent $(r=.93, n=168)$. The questionnaire result shows a high correlation with the perceived product satisfaction $\left(r_{s}=.86, p<.001, n=168\right)$. The questionnaire was rated as user-friendly and suitable for everyday use. The result of the questionnaire can be compared with objective values and considered when making purchasing decisions. 


\section{Identification of noise pollution in neonatal intensive care units based on work process analyses}

Ann-Kathrin Carl, Fachhochschule Münster, Steinfurt, Germany, a.carl@fh-muenster.de Niels Hinricher, Fachhochschule Münster, Steinfurt, Germany, niels.hinricher@fh-muensterde Lorenz Müller, Fachhochschule Münster, Steinfurt, Germany, lorenz.mueller@fh-muenster.de Claus Backhaus, Fachhochschule Münster, Steinfurt, Germany, claus.backhaus@fh-muenster.de

Each year in Germany, about 70.000 babies are born premature. The neonatal intensive care unit (NICU) ensures the survival of preterm infants. However, it simultaneously presents a great burden for the underdeveloped infant and his parents. A major problem is the high noise pollution in NICUs. Noise affects the development of auditory and communication skills, has direct influence on the cardiovascular and respiratory system and the sleeping behaviour of preterm infants. The aim of the study is to analyse the workflow of hospital staff and to identify systematic noise pollution in NICUs.

For this purpose, a contextual inquiry followed by a participative process analyses in NICUs are conducted. In addition, a video camera is placed in the working environment to record the work process of the hospital staff. Early, late and night shifts are analysed for one week each. Noise measurements and spectral analyses are carried out during the process analyses. A sound level meter is placed inside and one outside of the incubator. Additionally, heart rate, blood pressure and oxygen saturation of the preterm infants are extracted from the patient monitoring system. Particularly loud and onerous aspects of the daily work routine as well as medical devices are identified by analysing the physiological reaction of preterm infants to noise.

The expected results concern a systematic presentation and good transparency of working processes in NICUs. Furthermore, systematic noise sources concerning medical devices, and the hospital structure as well as the reaction of preterm infants to these sources are identified.

The study helps to create a sound-adjusted NICU to create an environment for preterm infants in which they can develop healthy and positive. 


\section{Usability assessment of patient hoists as a purchasing guide for health facilities}

Lorenz Müller, Fachhochschule Münster, Steinfurt, Germany, lorenz.mueller@fh-muenster.de Saskia Sobkowicz, Fachhochschule Münster, Steinfurt, Germany, s.sobkowicz@fh-muenster.de Elisabeth Ibenthal, Fachhochschule Münster, Steinfurt, Germany, e.ibenthal@ @fh-muenster.de Claus Backhaus, Fachhochschule Münster, Steinfurt, Germany, claus.backhaus@fh-muenster.de

Nurses suffer significantly more often from musculoskeletal injuries than other professions. A reason for this is the frequent mobilization of patients without assistive devices. Therefore, mobile patient hoists were developed to reduce physical strains. However, hoists are rarely used. A possible reason is a low user acceptance due to a poor usability of available hoists. To support health care facilities in purchasing ergonomically-designed hoists a comparative evaluation was carried out to identify design weaknesses. 12 hoists were tested by 40 nurses in a usability test. Every participant tested three patient hoists in randomized order. Scenarios were the mobilization of a dummy $(47 \mathrm{~kg})$ from a bed into a wheelchair, from a wheelchair onto a toilet and from the floor into a bed. Each scenario was based on 13 to 14 tasks. Every hoist was tested ten times. The effectiveness was measured by the success rate. To identify poorly designed components, success rates were clustered into five categories, which describe individual hoist parts. User acceptance was measured using a standardized questionnaire (16 items) in the range of 0 to 100 and an open survey. The success rate over all hoists ranges from $88 \%$ to $95 \%(\mathrm{M}=92 \%, \mathrm{SD}=2 \%)$. Serious use errors which were not corrected, or which led to an interruption of the work process occurred during $25 \%$ of the scenarios. The slingbars show the lowest success rate $(\mathrm{M}=82 \%, \mathrm{SD}=6 \%)$. One hoist's slingbar only reached a success rate of $68 \%$. The user acceptance of the hoists ranges from 49 to $86(\mathrm{M}=68$, $\mathrm{SD}=10$ ). The study identifies hoists with an above-average usability. Besides structural problems like limited space or a poor product availability, the detected deficits are a possible reason why current hoists are not used in everyday practice. 


\section{Patient and operating safety with electrical therapy beds - A systematic analysis of clamping and crushing risks}

Saskia Sobkowicz, FH Münster, Zentrum für Ergonomie und Medizintechnik, Steinfurt, Germany, s.sobkowicz@ fhmuenster.de

Elisabeth Ibenthal, FH Münster, Zentrum für Ergonomie und Medizintechnik, Steinfurt, Germany, e.ibenthal@ fhmuenster.de

Claus Backhaus, FH Münster, Zentrum für Ergonomie und Medizintechnik, Steinfurt, Germany, claus.backhaus@ fhmuenster.de

Electrical therapy beds differ in their lift mechanisms, operating elements and safety measures against accidental actuation of the height adjustment. In combination, these components result in different types of therapy beds. The electrically height-adjustment facilitates treatments of patients, but also represents hazards for employees and others. In the past, accidental actuation of the height adjustment led to serious clamping and crushing injuries, partially resulting in death. Hence, the Federal Institute for Drugs and Medical Devices in Germany recommends the equipment of therapy beds with safety measures, e.g. lockout boxes, since 2004. Despite an existing lockout box, a fatal accident occurred in 2017 during cleaning work.

In our investigation, the clamping and crushing risks of different therapy bed types will be analysed and evaluated. In a market analysis, representative types of therapy beds are determined. Subsequently, clamping and crushing risks of different therapy bed types are examined with fault tree analyses (DIN EN 61025). Damage-causing events are determined for each type and parsed into sub-events in a top-down analysis. For the first determination of events, injuries reported to the Federal Institute for Drugs and Medical Devices (Germany), the Food and Drug Administration (USA) and the German Critical Incident Reporting System are investigated. To quantify clamping and crushing risks, minimum cutsets and single point of failures are determined for each therapy bed type.

The market analysis produces an overview of lift mechanisms, operating elements and safety measures. The fault tree analyses result in hierarchical structures that analytically and systematically represent the interaction of events leading to a clamping or crushing injury. By comparing the minimum cutsets and single point of failures, a ranking of therapy bed types emerges for the risk of clamping and crushing injuries. Furthermore, recommendations for the prevention of such injuries are derived from the identified damage-causing events. 